\title{
Streptomycin Action and Anaerobiosis
}

\author{
By MARGOT KOGUT, J. W. LIGHTBOWN AND P. ISAACSON \\ Department of Biological Standards, National Institute for Medical Research, \\ London, N.W. 7
}

(Received 16 October 1964)

SUMMARY

The question was asked whether the insensitivity to dihydrostreptomycin and streptomycin exhibited by facultatively anaerobic organisms growing strictly anaerobically could be due entirely to the lack of an anaerobic mechanism for the uptake of these antibiotics. A technique is described here which allows exposure of Escherichia coli в to dihydrostreptomycin, under conditions which are known to promote its intracellular accumulation, for a time, followed by further incubation in a growth medium under aerobic as well as under strictly anaerobic conditions, after removal of the extracellular antibiotic. With this technique it was possible to make a quantitative comparison of the inhibitory effects of dihydrostreptomycin on aerobic and anaerobic growth of $\boldsymbol{E}$. coli B under conditions where no further uptake of the antibiotic could occur. The results show that for any given treatment of $E$. coli B with dihydrostreptomycin, subsequent aerobic and anaerobic growth rates are inhibited to exactly the same extent. It is concluded that the need for aerobic metabolism in the expression of antibiotic effect by dihydrostreptomycin is concerned only with its uptake into the organisms.

\section{INTRODUCTION}

In a review Kogut \& Lightbown (1964a) pointed out that the current hypothesis about the mode of action of streptomycin, namely that it inhibits protein synthesis (Erdös \& Ullmann, 1959; Hahn et al. 1962) by combining with the ribosomes of sensitive organisms (Spotts \& Stanier, 1961; Spotts, 1962; Flaks, Cox \& White, 1962; Speyer, Lengyel \& Basilio, 1962; Mager, Benedict \& Artman, 1962) leaves unexplained the frequently reported observation that strictly anaerobic bacteria and facultatively anaerobic bacteria when growing anaerobically are insensitive to the drug (Lightbown, 1957). However, Hancock (1962a) in a study of the intracellular accumulation of streptomycin, showed that no such accumulation took place when facultatively anaerobic organisms were grown under conditions which inhibited aerobic respiration. It seemed possible, therefore, that this dependence on aerobic metabolism of the uptake of streptomycin (and dihydrostreptomycin) into the organism might account for the necessity of aerobic conditions for antibiotic activity of the drug to be expressed. Since it is now known that several factors influence the uptake and intracellular accumulation of streptomycin (Hancock, $1962 a, b$; Hurwitz \& Rosano, 1962) and since such intracellular accumulation is presumably a necessary but by no means a sufficient condition for the antibiotic action, it seemed desirable to use experimental systems in which the entry of streptomycin into the cell could be dissociated from its further effects, to see 
whether the effect of anaerobiosis is solely on uptake. The present paper describes a technique for achieving this, and compares the growth-inhibitory effect of dihydrostreptomycin on a sensitive facultative bacterial strain during aerobic and anaerobic growth, subsequent to antibiotic treatments which are known to promote its intracellular accumulation by the organisms. Parts of this work have been communicated previously (Kogut \& Lightbown, 1963; Kogut \& Lightbown, 1964b).

\section{METHODS}

Organism and growth conditions. The organism used was Escherichia coli в (obtained from Dr M. Pollock, National Institute for Medical Research) which was kept at $4^{\circ}$ on Hedley Wright agar slopes (Wright, 1933) after growth for $18 \mathrm{hr}$ at $37^{\circ}$, and subcultured weekly. For experiments the growth medium was that of McQuillen \& Roberts (1954) modified by the addition before autoclaving of $0.2 \%$ $(\mathrm{w} / \mathrm{v})$ trisodium citrate. A $10 \%(\mathrm{w} / \mathrm{v})$ glucose solution (sterilized by filtration) was added at the time of inoculation to give a final concentration of $0 \cdot 2 \%(\mathrm{w} / \mathrm{v})$ glucose. The temperature of incubation was $32^{\circ}$. Growth was measured as extinction $(E)$ at $500 \mathrm{~m} \mu\left(E_{500}\right)$ in a Hilger 'Uvispeck' spectrophotometer with optical cells of $5 \mathrm{~mm}$. light path, and recorded as logarithms to the base 2 (Finney, Hazlewood \& Smith, 1955). Standard inocula were prepared as follows. The growth from one agar slope was suspended in $1 \mathrm{ml}$. of sterile growth medium, and $0.2 \mathrm{ml}$. of this suspension added to $50 \mathrm{ml}$. sterile medium in a $250 \mathrm{ml}$. Erlenmeyer flask. This inoculum was allowed to grow at $32^{\circ}$ for $6-10 \mathrm{hr}$, or another logarithmically growing culture was used. The extinction $\left(E_{500}\right)$ was measured, and a portion of the culture diluted with medium to a calculated $E_{500}$ of 0.001 . Of this suspension, $1.0 \mathrm{ml} . / 50 \mathrm{ml}$. fresh growth medium gave cultures in the logarithmic phase after incubation for 16-18 hr at $32^{\circ}$. Aerobiosis was achieved by shaking flasks in a constant temperature waterbath at 120 strokes $/ \mathrm{min}$. (throw $4.5 \mathrm{~cm}$.); under these conditions it was determined experimentally that up to $60 \mathrm{ml}$. culture in a $250 \mathrm{ml}$. Erlenmeyer flask, and up to $300 \mathrm{ml}$. in a $1 \mathrm{l}$. Erlenmeyer flask gave adequate aeration.

Strictly anaerobic growth under conditions where samples could be withdrawn for $E_{500}$ measurements without admitting air to the culture, was achieved as follows (Fig. 1). The needle of a $30 \mathrm{ml}$. all-glass hypodermic syringe was replaced with a length of capillary tubing (glass and silicone rubber) and the end of this tubing was closed with a spring clip. The assembled apparatus, wrapped in paper, was sterilized by autoclaving $\left(20 \mathrm{~min} ., 121^{\circ}\right)$. When needed, the plunger was withdrawn, the culture introduced into the barrel, the plunger replaced and all gas expelled until the capillary tubing was filled with culture. The filled syringes were held in the waterbath by means of spring clips mounted on a board so that the whole of the culture in the syringe and most of that in the capillary tubing was immersed. Samples, when necessary, were withdrawn by depressing the plunger and opening the capillary tubing. The first $\mathbf{0 . 5} \mathrm{ml}$. of each sample was always discarded. Anaerobic additions to such cultures could be made by injection through the silicone tubing into the barrel of the syringe from another syringe; mixing was achieved by drawing the culture into the injecting syringe a number of times.

Total counts were made by using a Thoma counting chamber of depth $\mathbf{0 . 0 2} \mathrm{mm}$.; a minimum of 400 organisms/sample were counted. 
Dry weights were determined by drying appropriate samples, after washing 3 times with water by centrifugation, in aluminium foil 'boats' on a hot plate at $60^{\circ}$ and leaving in a desiccator over $\mathbf{P}_{2} \mathrm{O}_{5}$ until the weights were constant.

Filtration treatment. Rapid transfer of growing organisms from one medium to another was achieved by filtration on membrane filters (Millipore; diameter $47 \mathrm{~mm}$.; A.P.D., $0 \cdot 22 \mu$ ) followed by washing with and resuspension in fresh medium prewarmed to the growth temperature.

Assays of dihydrostreptomycin concentrations were by the diffusion method (Humphrey \& Lightbown, 1952). Dihydrostreptomycin sulphate, potency 800 i.u./ mg. was obtained from Distillers (Biochemicals) Co. Ltd. Speke, Liverpool 1.

Chemicals used were all A.R. grade.

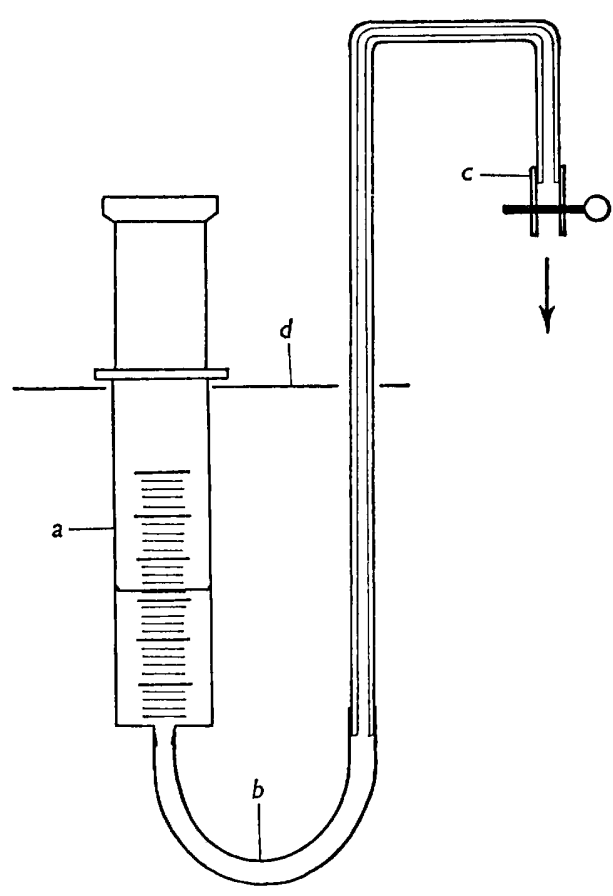

Fig. 1

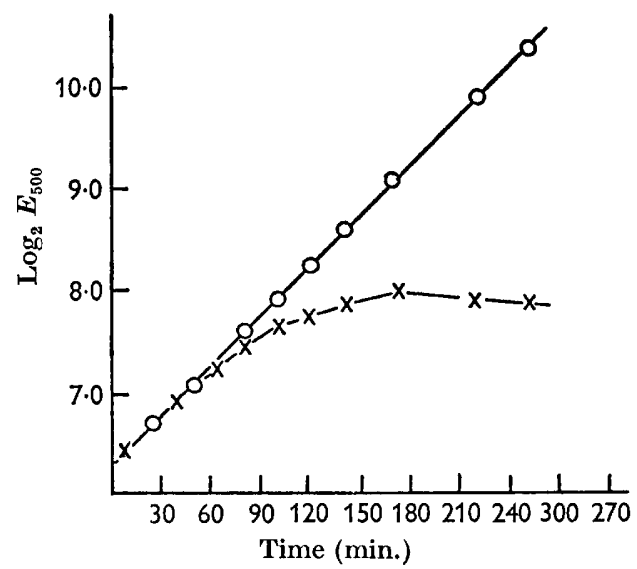

Fig. 2

Fig. 1. Syringe for anaerobic growth of E. coli B. (a) $30 \mathrm{ml}$. all glass-syringe half filled with culture. (b) Silicone rubber tubing connecting syringe with capillary glass tubing. (c) Silicone rubber tubing carrying spring-clip for withdrawing samples. $(d)$ Level of water bath relative to barrel of syringe and capillary tubing.

Fig. 2. Growth curve of $E$. coli $\mathrm{B}$, without, and after addition of, $20 \mu \mathrm{g}$. $/ \mathrm{ml}$. dihydrostreptomycin. Growth medium and temperature as described in 'Methods'. $\boldsymbol{E}_{500}$ in cuvettes of $5 \mathrm{~mm}$. light path, plotted as $\log _{2}$ on the ordinate. $\mathrm{O}-\mathrm{O}=$ Control. $x-x=+20 \mu \mathrm{g}$. dihydrostreptomycin sulphate $/ \mathrm{ml}$. added at $0 \mathrm{~min}$.

\section{RESULTS}

Under the experimental conditions described the specific growth rates, $\mu$ (Herbert, Elsworth \& Telling, 1956) of Escherichia coli B were approximately $1.0(\mu=$ doublings/hr). Addition of $20 \mu \mathrm{g}$. dihydrostreptomycin sulphate/ml. culture 
during the early exponential stage of aerobic growth $\left(E_{500} 0 \cdot 1-0 \cdot 2\right)$ produced a time course of growth inhibition as shown in Fig. 2. Table 1 shows the relationships between $E_{500}$ measurements and total counts and dry-weight determinations for samples taken at different times, with and without dihydrostreptomycin. The results show that there was essentially no change in the ratios $E_{500}$ :dry-weight and total count up to the time when growth inhibition was complete, thus justifying the use of $E_{500}$ determinations to measure inhibition of growth.

Table 1. Ratio of total counts and dry weights to $E_{500}$ of $E$. coli в cultures growing in the presence and absence of dihydrostreptomycin

\begin{tabular}{|c|c|c|c|c|c|c|c|c|c|}
\hline \multirow[b]{3}{*}{$\begin{array}{l}\text { Expt. } \\
\text { no. }\end{array}$} & \multirow{2}{*}{\multicolumn{4}{|c|}{ Controls }} & \multicolumn{5}{|c|}{$+20 \mu \mathrm{g}$. dihydrostreptomycin sulphate $/ \mathrm{ml}$. at $0 \mathrm{~min}$. } \\
\hline & & & & & & & $\%$ & & \\
\hline & $\begin{array}{l}\text { Time } \\
\text { (min.) }\end{array}$ & $E_{500}$ & $\begin{array}{c}\text { Total count } / \\
E_{500}\end{array}$ & mg. dry wt./ & $\begin{array}{l}\text { Time } \\
\text { (min.) }\end{array}$ & $E_{500}$ & $\begin{array}{c}\text { Inhibition } \\
\text { of } \mu\end{array}$ & $\begin{array}{c}\text { Total count } / \\
E_{500}\end{array}$ & mg. dry wt./ \\
\hline \multirow[t]{8}{*}{1} & 15 & $0 \cdot 115$ & $1.74 \times 10^{9}$ & - & 15 & $0 \cdot 116$ & $\mathbf{0}$ & $1.78 \times 10^{9}$ & - \\
\hline & 30 & 0.136 & $1.66 \times 10^{9}$ & - & 45 & $0 \cdot 161$ & 0 & $1.93 \times 10^{9}$ & - \\
\hline & 60 & $0 \cdot 192$ & $1.70 \times 10^{9}$ & - & 60 & $0 \cdot 186$ & 17 & $1.72 \times 10^{9}$ & - \\
\hline & 85 & $0 \cdot 251$ & $2.08 \times 10^{9}$ & - & 95 & $0 \cdot 240$ & 45 & $2.07 \times 10^{9}$ & $0 \cdot 530$ \\
\hline & 110 & 0.349 & - & 0.565 & 110 & $0 \cdot 258$ & 60 & $1.68 \times 10^{9}$ & - \\
\hline & 115 & $0 \cdot 349$ & $1.68 \times 10^{9}$ & - & 130 & 0.281 & 64 & $1.80 \times 10^{9}$ & - \\
\hline & 185 & 0.740 & - & $0 \cdot 565$ & 145 & 0.274 & 92 & $2.02 \times 10^{9}$ & - \\
\hline & & & & & 162 & $0 \cdot 287$ & 96 & $1.94 \times 10^{9}$ & - \\
\hline \multirow[t]{5}{*}{2} & 46 & $0 \cdot 227$ & $1.84 \times 10^{9}$ & - & 30 & $0 \cdot 185$ & 0 & $1.55 \times 10^{9}$ & - \\
\hline & 85 & 0.349 & $1.41 \times 10^{9}$ & - & 60 & $0 \cdot 246$ & 13 & $1 \cdot 44 \times 10^{9}$ & - \\
\hline & 120 & 0.540 & $1 \cdot 64 \times 10^{9}$ & - & 85 & $0 \cdot 309$ & 29 & $1.86 \times 10^{9}$ & 0.563 \\
\hline & 193 & $1 \cdot 164$ & $1.67 \times 10^{9}$ & 0.590 & 100 & $0 \cdot 340$ & $42 \cdot 5$ & $2.17 \times 10^{9}$ & - \\
\hline & & & & & 120 & $0 \cdot 375$ & 56 & $1.67 \times 10^{9}$ & $0 \cdot 555$ \\
\hline \multirow[t]{6}{*}{3} & 20 & $0 \cdot 101$ & $1.83 \times 10^{9}$ & - & 80 & $0 \cdot 241$ & 48 & - & 0.526 \\
\hline & 185 & 0.990 & - & 0.560 & 130 & 0.288 & 88 & $1.58 \times 10^{9}$ & 0.545 \\
\hline & & & & & 145 & $0 \cdot 290$ & 100 & $1.41 \times 10^{9}$ & - \\
\hline & & & & & 165 & $0 \cdot 291$ & 100 & $1.36 \times 10^{9}$ & - \\
\hline & & & & & 185 & $0 \cdot 294$ & 100 & $1.37 \times 10^{9}$ & - \\
\hline & & & & & 255 & $0 \cdot 260$ & 100 & $1.45 \times 10^{9}$ & 一 \\
\hline
\end{tabular}

In preliminary experiments a culture of Escherichia coli $\mathbf{B}$ growing aerobically in the early logarithmic phase, was divided into two flasks, A and B. From flask A, two samples each of $30 \mathrm{ml}$. were withdrawn and placed in syringes for anaerobic growth, at the same time as dihydrostreptomycin sulphate $20 \mu \mathrm{g} . / \mathrm{ml}$. was added to the flask B. Of the two anaerobic controls, one received no antibiotic, and to the other dihydrostreptomycin sulphate $20 \mu \mathrm{g}$. $/ \mathrm{ml}$. was added, by injecting it through the silicone rubber tube, at a time when it was estimated from $Q_{\mathrm{O}_{2}}$ and extinction measurements that anaerobic growth should have been established. From flask B, samples were withdrawn at various times after the addition of dihydrostreptomycin and placed into syringes to grow anaerobically. Figure $3 a$ shows that when dihydrostreptomycin was added to a culture growing anaerobically, growth was unaffected. However (Fig. $3 b$ ) when a culture was made anaerobic at various times after the addition of dihydrostreptomycin, growth continued apparently exponentially, though at rates which were slower as compared with the anaerobic controls. The extent of inhibition appeared to be proportional to the duration of previous aerobic growth in the presence of dihydrostreptomycin. 
There were, however, difficulties in the quantitative interpretation of these results. First, at the low organism concentrations used, it always took 10-20 min. for the organisms to exhaust the dissolved oxygen in the medium and thus to establish strictly anaerobic conditions; during this period further uptake of dihydrostreptomycin might have occurred. Secondly, and more important, there was always a lag period during which no change in extinction occurred, before anaerobic growth became established (see Fig. $3 a, b$ ). It might be argued therefore that the

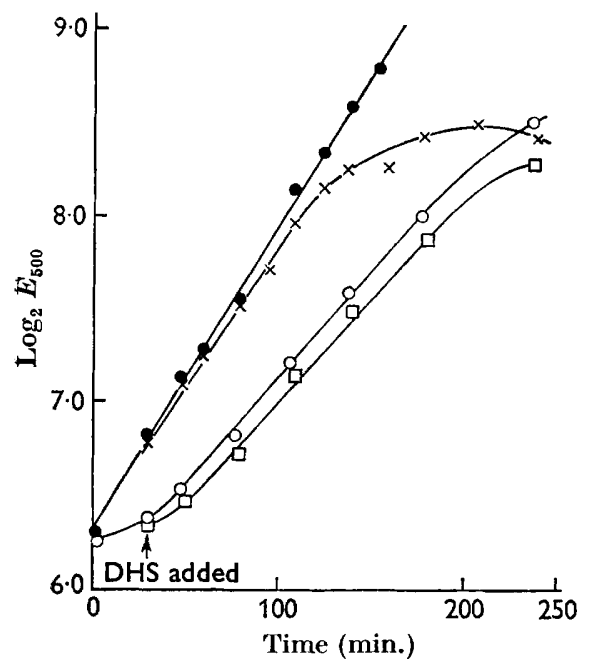

(a)

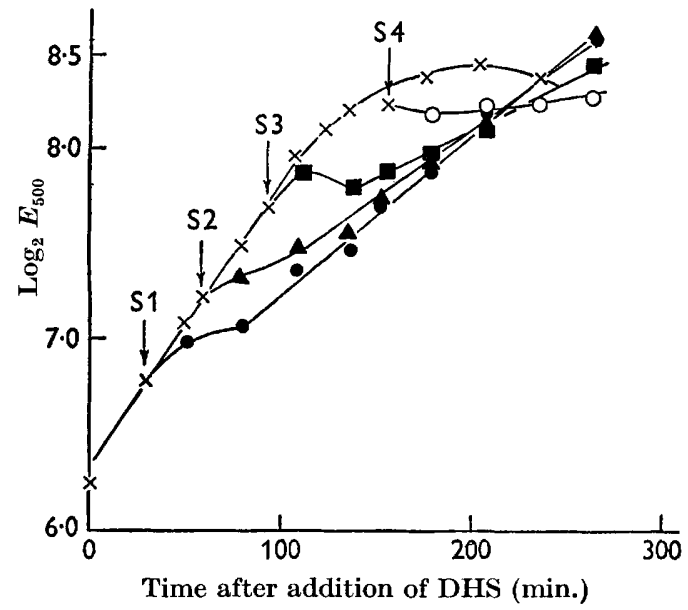

(b)

Fig. 3. Aerobic and anaerobic growth of Escherichia coli $\mathrm{B}$ after addition of $20 \mu \mathrm{g}$. dihydrostreptomycin sulphate/ml. (DHS). Growth medium and temperature as in 'Methods'. (a) - = Control growing aerobically. $\times-\times=$ Aerobic growth after addition of dihydrostreptomycin at $0 \mathrm{~min}$. $\mathrm{O}-\mathrm{O}=$ Sample removed from control at $0 \mathrm{~min}$. and transferred to anaerobic growth. $\square-\square=$ Sample removed from control at 0 min., transferred to anaerobic growth and dihydrostreptomycin added at arrow. $(b) \times-\times=$ Parent culture growing aerobically in presence of dihydrostreptomycin added at 0 min. $-0=S 1, \Delta-\Delta=S 2, \quad \square-\square=S 3, \quad O-O=S 4$, samples removed at various times after addition of dihydrostreptomycin, indicated by arrows, from aerobic parent culture, and transferred to anaerobic conditions.

lower rates of anaerobic growth, after treatment of the organisms with dihydrostreptomycin under aerobic conditions, might have been due to an inhibitory effect of the antibiotic, inside the organisms, on an adaptive process required to allow anaerobic growth (Fowler, 1951) and not to an effect on anaerobic growth itself. This possibility was eliminated by growing the organisms anaerobically for two generations or more before making them aerobic and adding the dihydrostreptomycin. After such a period of previous anaerobic growth, followed by a limited period of aerobic growth in the presence of dihydrostreptomycin, the next changeover to anaerobiosis occurred without a lag in the growth curve.

A further modification of the experimental technique made possible a direct quantitative comparison between the degree of inhibition of aerobic and anaerobic growth. Samples withdrawn from a parent culture growing aerobically in the 
presence of dihydrostreptomycin were filtered on membranes, these washed twice with the original volume of pre-warmed antibiotic-free growth medium to remove the free as well as most of the adsorbed dihydrostreptomycin (Hancock, $1962 a$;

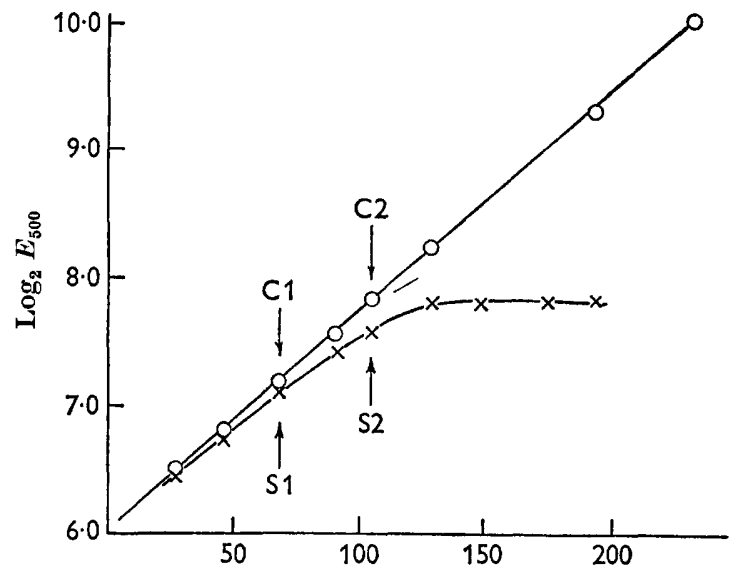

Time after addition of DHS (min)

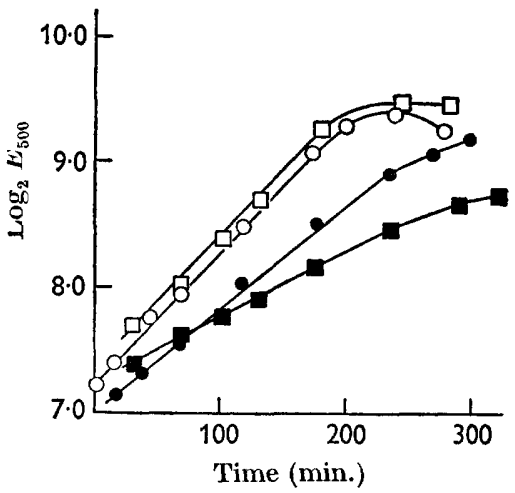

(b)

(a)

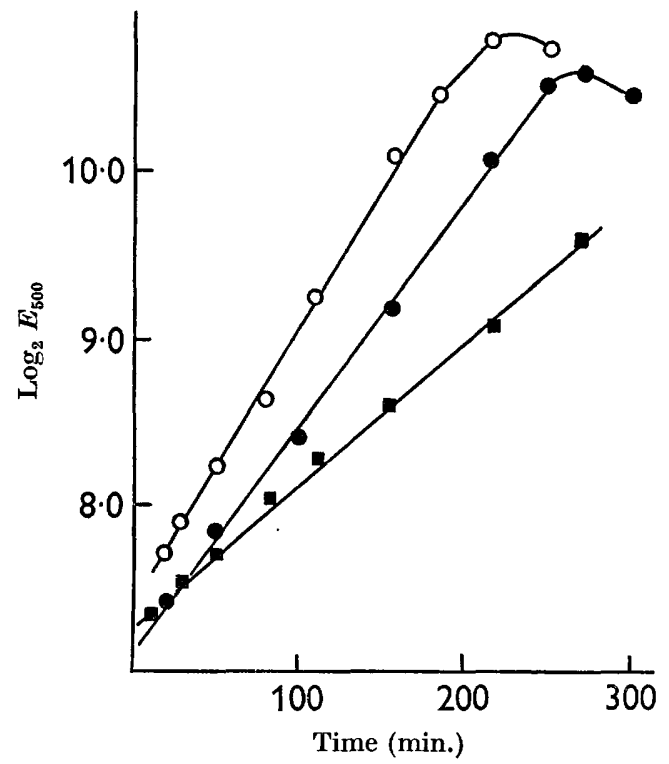

(c)

Fig. 4. Effect of $20 \mu \mathrm{g}$. dihydrostreptomycin sulphate $/ \mathrm{ml}$. during aerobic exponential growth on subsequent aerobic and anaerobic growth in the absence of extracellular antibiotic. (a) Parent cultures: $\mathrm{O}-\mathrm{O}=$ control, $\mathrm{x}-\mathrm{x}=+$ dihydrostreptomycin. (b) Anaerobic growth of samples removed from parent cultures at times indicated by arrows (in $(a))$ and freed of extracellular antibiotic by filtration and washing. Open symbols $=$ controls, filled symbols = treated culture; - -,$O-O=S 1 ; \square-\square, \square-\square=S 2$. (c) Aerobic growth of samples removed from parent cultures at times indicated by arrows (in $(a)$ ) and freed of extracellular antibiotic by filtration and washing. Open symbols $=$ control, filled symbols = treated culture; $-0,0-0=S 1 ; \mathbf{O}-\mathbf{0}=\mathbf{S} 2$. 
Hurwitz \& Rosano, 1962) and then the organisms resuspended in the original volume of warm antibiotic-free medium. Each resuspended sample was divided into two equal portions of which one was grown aerobically and the other anaerobically. Sensitive antibiotic assays of the washings and resuspending medium, collected separately, showed that less than $0 \cdot 10 \mu \mathrm{g}$. free dihydrostreptomycin $/ \mathrm{ml}$. was carried over into the final resuspended culture. The time taken from withdrawal

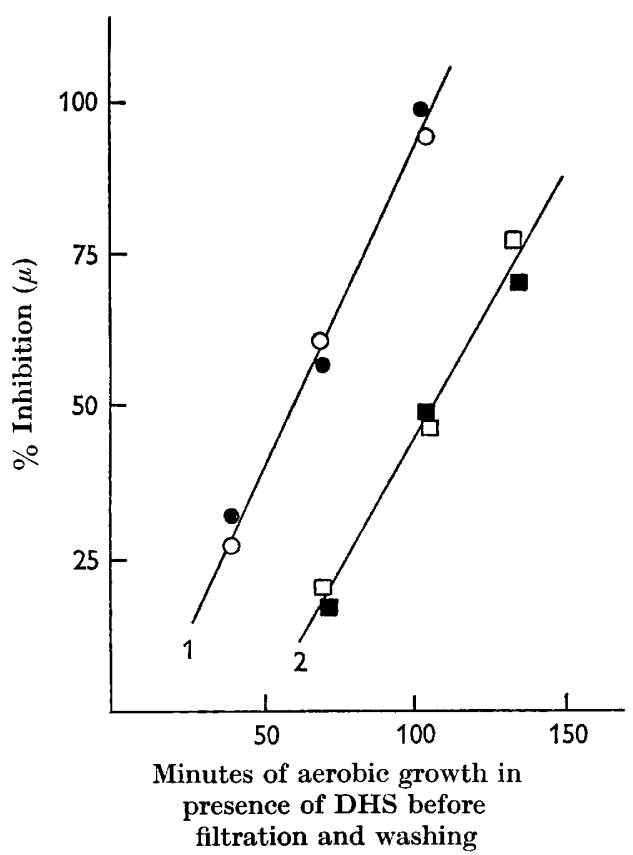

(a)

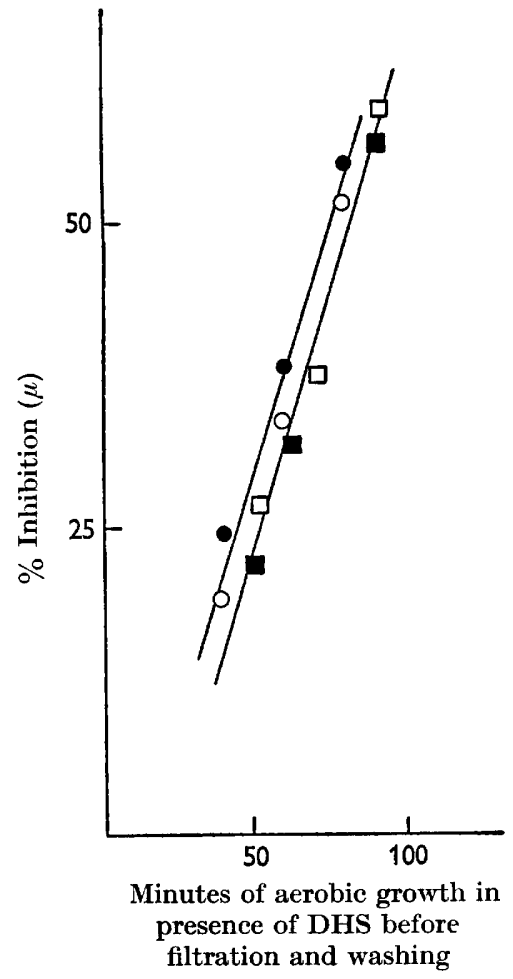

(b)

Fig. 5. Percentage inhibition of aerobic and anaerobic growth rates of $E$. coli $\mathrm{B}$ as a function of duration of prior aerobic growth in different concentrations of dihydrostreptomycin. (a) $20 \mu \mathrm{g}$. dihydrostreptomycin sulphate/ml. Two separate experiments : (1) without prior anaerobic growth, (2) addition of drug to aerobic culture after two generations of anaerobic growth. Open symbols = inhibition of aerobic growth, filled symbols = inhibition of anaerobic growth. (b) $100 \mu \mathrm{g}$. dihydrostreptomycin sulphate/ml., added to aerobic cultures after two generations of anaerobic growth. Two separate experiments represented by circles and squares. Open symbols = inhibition of aerobic growth, filled symbols = inhibition of anaerobic growth.

of the sample for filtration to replacement of the culture in the water bath, was 5-10 min. A typical experiment is depicted in Fig. 4. Dihydrostreptomycin-free controls, which were also subjected to the 'filtration treatment' gave consistent aerobic growth rates for samples filtered at various times, and only one such sample was therefore taken as control for aerobic growth (Fig. 4c). Anaerobically, however, the growth rates of the controls sometimes varied with the duration of previous aerobic growth. Therefore samples for filtration were taken from the controls each time a sample was taken from the dihydrostreptomycin-treated cultures (Fig. $4 b$ ). 
In a large number of similar experiments, it was always found that samples withdrawn from the dihydrostreptomycin-treated culture before growth had become completely inhibited, continued to grow, both aerobically and anaerobically, but at rates which were slower in proportion to the duration of the prior aerobic growth in dihydrostreptomycin and to the initial concentration of dihydrostreptomycin in the medium.

As the growth of all filtered samples, both aerobically and anaerobically, appeared to be exponential, it was possible to make a quantitative comparison of inhibition of aerobic and anaerobic growth on the basis of the different growth rates. Figure 5 shows the $\%$ inhibition of aerobic and anaerobic growth rates plotted against the duration of the previous aerobic growth in presence of dihydrostreptomycin. These results are from four separate experiments, of the kind illustrated in Fig. 4. The cycle of anaerobic growth before treatment with dihydrostreptomycin, which was introduced in order to abolish the lag period which occurred on changing from aerobic to anaerobic growth, tended to decrease the sensitivity of the organism to dihydrostreptomycin; i.e. it took longer to reach a given degree of inhibition for a given antibiotic concentration. The 'anaerobic lag' gradually returned when cultures were incubated aerobically, and was occasionally apparent in experiments of a duration necessary to obtain a relatively high degree of inhibition with dihydrostreptomycin sulphate $20 \mu \mathrm{g}$. $/ \mathrm{ml}$. In some experiments, therefore, the concentration of dihydrostreptomycin sulphate was increased to $100 \mu \mathrm{g} . / \mathrm{ml}$. so as to obtain approximately $\mathbf{5 0} \%$ inhibition of growth rate in $\mathbf{3 0}$ min. or less (Fig. $\mathbf{5 b}$ ). In all cases, the \% inhibition of growth rate, in terms of the appropriate control, was the same for aerobic and anaerobic growth, and appeared to be a function of the duration of treatment with dihydrostreptomycin, and of its initial concentration in the culture medium.

\section{DISCUSSION}

Escherichia coli в when growing exponentially under anaerobic conditions was not inhibited by dihydrostreptomycin; this confirmed previous observations with other streptomycin-sensitive organisms. In two types of further experiments cultures of $E$. coli в were exposed to dihydrostreptomycin under conditions which lead to a continued uptake of the antibiotic with an increasing degree of inhibition (Hancock, $1962 b$ ). When samples of such cultures were removed at different degrees of inhibition and incubated under anaerobic conditions, growth continued at a slower rate, thus indicating that the effects of dihydrostreptomycin on growth, once established, were not dependent on aerobic metabolism. In the first series of experiments it was impossible to compare the degrees of inhibition of aerobic and anaerobic growth produced by a given concentration of dihydrostreptomycin. Aerobically the inhibition was progressive, whereas anaerobically the increase in inhibition was arrested at a value related to the degree of inhibition existing at the time when conditions were changed from aerobic to anaerobic. The findings of Hancock $(\mathbf{1 9 6 2} a)$ that anaerobic conditions prevented uptake of streptomycin, whereas under aerobic conditions exponentially growing organisms accumulated streptomycin, offer an explanation of this phenomenon.

The use of the filtration technique in the second series of experiments enabled organisms to be exposed to dihydrostreptomycin for a given time and then further 
accumulation prevented by transfer to dihydrostreptomycin-free medium. Such organisms grew exponentially at a slower rate, aerobically and anaerobically. Under these conditions the degree of inhibition in the two states was compared directly and was found to be the same. The lack of dependence of established inhibition of growth rate on aerobic metabolism was found over a wide range of degrees of inhibition and it seems quite clear, therefore, that the need for aerobic metabolism is concerned only with uptake of dihydrostreptomycin. It is possible that the linear relationship between previous duration of growth in the presence of dihydrostreptomycin, and \% inhibition of growth rate, reflects a linear relationship between intracellular concentration of dihydrostreptomycin and the growth rate. If it could be shown that the intracellular concentration of dihydrostreptomycin was a linear function of time of exposure, this interpretation would seem reasonable. Although Litwak \& Pramer (1957) with Nitella, and Hancock (1962a) with several bacterial species showed that uptake of streptomycin was an energy-dependent process, and was affected by a variety of conditions, no adequate quantitative data on rates of uptake are available.

The difference in rate of development of inhibition between Escherichia coli в organisms previously exposed to anaerobic conditions, and those not so exposed, is probably due to an effect on the uptake of dihydrostreptomycin; it illustrates again the susceptibility of 'streptomycin' effects to different environmental conditions. The experimental technique described here enabled the uptake of dihydrostreptomycin to be stopped at any desired level, and organisms with different fixed doses of dihydrostreptomycin could then be examined and compared. The inhibited exponential growth of such organisms with subsequent recovery has not been previously described; it is examined more fully in a later paper.

It would seem to be a naïve reflexion that growth-inhibitory substances, in order to manifest their growth-inhibitory effects, will in the majority of cases have to pass through or get inside, at least the outermost layer of the organism. Such processes should be regarded as 'uptake into the organism'. Although with nutrients and metabolites such 'uptake' is no longer thought to be simple diffusion or penetration of a porous surface, it is surprising how little attention has been devoted to the character and mechanism of 'uptake' of antibiotics. Since this is a necessary but presumably quite separate pre-condition for the actual growth-inhibitory effects, a better understanding of the relationship between these two phases of antibiotic action may be required.

Our thanks are due to Mr M. Wilkins for conscientious and willing technical assistance.

\section{REFERENCES}

Erdös, T. \& Ullmann, A. (1959). Efiect of streptomycin on the incorporation of aminoacids labelled with carbon-14 into ribonucleic acid and protein in a cell-free system of a Mycobacterium. Nature, Lond., 183, 618.

Finney, D. J., Hazlewood, T. \& Smith, M. J. (1955). Logarithms to base two. J. gen. Microbiol. 12, 222.

Flaks, J. G., Cox, E. C. \& White, J. R. (1962). Inhibition of polypeptide synthesis by streptomycin. Biochem. Biophys. Res. Commun. 7, 385.

Fowler, B. (1951). The relationship between fermentation and enzyme adaptation. Biochim. biophys. Acta, 7, 563. 


\section{4}

Hahn, F. E., Ciak, J., Wolfe, A. D., Hartman, R. E., Allison, J. L. \& Hartman, R. S. (1962). Studies on the mode of action of streptomycin. II. Effects of streptomycin on the synthesis of proteins and nucleic acids and on cellular multiplication in Escherichia coli. Biochim. biophys. Acta, 61, 741.

HaNCock, R. $(1962 a)$. Uptake of ${ }^{14} \mathrm{C}$-streptomycin by some micro-organisms and its relation to their streptomycin sensitivity. J. gen. Microbiol. $28,493$.

Hancock, R. $(1962 b)$. Uptake of ${ }^{14} \mathrm{C}$-streptomycin by Bacillus megaterium. J. gen. Microbiol. 28, 503.

Herbert, D., Elsworth, R. \& Telling, R. C. (1956). The continuous culture of bacteria; a theoretical and experimental study. J. gen. Microbiol. 14, 601.

Humphrey, J. H. \& Lightbown, J. W. (1952). A general theory for plate assay of antibiotics with some practical applications. J. gen. Microbiol. 7, 129.

Hurwitz, C. \& Rosano, C. L. (1962). Accumulation of label from ${ }^{14} \mathrm{C}$-streptomycin by Escherichia coli. J. Bact. 83, 1193.

Kogut, M. \& Lightbown, J. W. (1963). Streptomycin action and aerobiosis. Biochem. J. 89, $18 P$.

Kogut, M. \& Lightвown, J. W. (1964a). The mode of action of streptomycin. In Experimental Chemotherapy, vol. 3, Ed. by R. J. Schnitzer \& F. Hawking. New York: Academic Press Inc.

Kogut, M. \& Ligmtbown, J. W. (1964b). Growth of dihydrostreptomycin-treated Escherichia coli after removal of extracellular antibiotic. J. gen. Microbiol. 34, x.

Lighтвоw, J. W. (1957). Metabolic processes underlying streptomycin resistance. G. ital. chemioterap., 4, 22.

Litwak, G. \& Pramer, D. (1957). Absorption of antibiotics by plant cells. III. Kinetics of streptomycin uptake. Arch. Biochem. Biophys. 68, 396.

Mager, J., Benedict, M. \& Artman, M. (1962). A common site of action for polyamines and streptomycin. Biochim. biophys. Acta, 62, 202.

MCQuillen, K. \& Roberts, R. B. (1954). The utilization of acetate for synthesis in Escherichia coli. J. biol. Chem. 207, 81.

Speyer, J. F., Lengyel, P. \& Basilio, C. (1962). Ribosomal localization of streptomycin sensitivity. Proc. natn. Acad. Sci. U.S.A. 48, 684.

Spotts, C. R. \& Stanier, R. Y. (1961). Mechanism of streptomycin action on bacteria: a unitary hypothesis. Nature, Lond., 192, 633.

Spotts, C. R. (1962). Physiological and biochemical studies on streptomycin dependence in Escherichia coli. J. gen. Microbiol. 28, 347.

Wright, H. D. (1933). Peptone culture medium. J. Path. Bact. 37, 257. 